\title{
What Is Half a Neutrino? Reviewing Cosmological Constraints on Neutrinos and Dark Radiation
}

\author{
S. Riemer-Sørensen ${ }^{1,2}$, D. Parkinson ${ }^{1}$ and T. M. Davis ${ }^{1}$ \\ ${ }^{1}$ School of Mathematics and Physics, University of Queensland, Brisbane 4072, Queensland, Australia \\ ${ }^{2}$ Corresponding author. Email: signe@ physics.uq.edu.au
}

(Received January 23, 2013; Accepted January 31, 2013; OnLine Publication March 20, 2013)

\begin{abstract}
Neutrinos are one of the major puzzles in modern physics. Despite measurements of mass differences, the Standard Model of particle physics describes them as exactly massless. Additionally, recent measurements from both particle physics experiments and cosmology indicate the existence of more than the three Standard Model species. Here, we review the cosmological evidence and its possible interpretations.
\end{abstract}

Keywords: cosmological parameters - cosmology: observations - early Universe - elementary particles - neutrinos

\section{INTRODUCTION}

Neutrinos are the lightest of the known massive particles and one of the least understood. The cosmic neutrino background has a number density of $112 \mathrm{~cm}^{-3}$ (per species), which means that billions of neutrinos pass through our bodies every second, so they are by no means rare. Yet we do not know their basic properties, such as their mass and how many species there are.

\subsection{Particle physics neutrinos}

The discovery of the Higgs boson at the Large Hadron Collider (LHC) consolidates the power of the Standard Model of particle physics (Aad et al. 2012; Chatrchyan et al. 2012). However, there are still unsolved issues in the neutrino sector, with their masses remaining unexplained and uncertainty about the actual number of species.

The Standard Model contains three species of massless neutrinos that interact only through the weak interaction. By measuring the decay width of the $\mathrm{Z}^{0}$ boson the Large Electron Positron Collider (LEP) measured the number of weakly interacting neutrinos to be $N_{v}=2.92 \pm 0.05$ (Beringer et al. 2012), corresponding to the three generations of leptons, namely the electron, the muon and the tau particles.

Observations of neutrino disappearance, e.g. from $v_{e}$ sources (Cleveland et al. 1998; Fukuda et al. 1998), and their appearance, e.g. in $v_{\mu}$ beams (Abe et al. 2011), have led to the framework of neutrino mixing/oscillations, where the interaction eigenstates $\left(v_{e}, v_{\mu}, v_{\tau}\right)$ are described as su- perpositions of mass eigenstates $\left(v_{1}, v_{2}, v_{3}\right)$. Global fits to data from experiments using solar, atmospheric and reactor neutrinos have measured mass differences between the three species to be $\Delta m_{32}^{2}=\left|\left(2.43_{-0.08}^{+0.12}\right) \times 10^{-3}\right| \mathrm{eV}^{2}$ and $\Delta m_{21}^{2}=(7.50 \pm 0.20) \times 10^{-5} \mathrm{eV}^{2}$ (Beringer et al. 2012), thus requiring at least two mass eigenstates to have non-zero masses.

From studying the end-point of the electron energy distribution in $\beta$-decays, the Mainz and Troitsk experiments have limited the electron neutrino to have a mass smaller than 2.3 and $2.05 \mathrm{eV}$, respectively (95\% confidence level; Kraus et al. 2005; Aseev et al. 2011). For the specific case of neutrinos being Majorana particles (i.e. their own antiparticles), the Heidelberg-Moscow experiment has limited the effective neutrino mass to be less than $0.35 \mathrm{eV}$ (90\% confidence level) using the neutrinoless double $\beta$-decay (Klapdor-Kleingrothaus \& Krivosheina 2006) with similar results from EXO (Auger et al. 2012). However, no presentday experiment has the sensitivity to measure the absolute neutrino mass. The upcoming KArlsruhe TRItium Neutrino (KATRIN) experiment is expected to improve the sensitivity to $0.2 \mathrm{eV}$ (90\% confidence level) from $\beta$-spectroscopy, but this upper limit remains far above the observed mass differences.

The sign of $\Delta m_{32}$ is unknown, which means that we cannot know whether the ordering of the mass states is $m_{1}<m_{2} \ll$ $m_{3}$, similar to the leptons, where $m_{e}<m_{\mu} \ll m_{\tau}$. This is called normal hierarchy. If $m_{3} \ll m_{1}<m_{2}$, the scenario is called inverted hierarchy. 
For the short baseline (SBL) oscillations, there are some tensions between the various experiments allowing for, or even preferring, the existence of additional neutrino species that do not interact through the weak force, and are consequently denoted sterile. Depending on the exact analysis, the preferred scenario includes either one or two sterile neutrinos in addition to the three normal ones $(3+1$ and $3+2)$, with sterile neutrino masses on the order of $1 \mathrm{eV}$ (Kopp, Maltoni, \& Schwetz 2011; Mention et al. 2011; Huber 2011; Giunti \& Laveder 2011).

\subsection{Cosmological neutrinos}

Observations of the cosmic microwave background (CMB) have, since the five-year data release from the Wilkinson $\mathrm{Mi}$ crowave Anisotropy Probe (WMAP), displayed a mild preference for an excess of relativistic energy density at the time of decoupling on top of what is provided by the photon density and three Standard Model neutrinos (Komatsu et al. 2009). This extra radiation has been dubbed 'dark radiation' and is often parametrised in terms of extra neutrino species such that $N_{\text {eff }}=N_{v}+\Delta N$. The most striking result is from the Atacama Cosmology Telescope (ACT) of $N_{\text {eff }}=5.3 \pm 1.3$ (Dunkley et al. 2011), which is shown in Figure 1 together with a selection of cosmological analyses from the literature. There is a clear trend favouring $N_{\text {eff }}>3$ across various models and data sets.

Note that $N_{\text {eff }}$ is often referred to as the effective number of neutrino species or the number of relativistic species, but basically it is a parametrisation of anything that would change the expansion rate at the time of decoupling. Consequently, fractional values of $N_{\text {eff }}$ do not actually require a non-integer number of neutrino species. ${ }^{1}$ Possibilities include other exotic particles such as axions (e.g. Melchiorri, Mena, \& Slosar 2007; Nakayama, Takahashi, \& Yanagida 2011; Erken et al. 2012), decaying particles or fields (Ichikawa et al. 2007; Nakayama et al. 2011; Fischler \& Meyers 2011; Boehm, Dolan, \& McCabe, 2012), gravity waves (Smith, Pierpaoli, \& Kamionkowski 2006), extra dimensions (Flambaum \& Shuryak 2006), and early dark energy (Calabrese et al. 2011; Gagnon \& Lesgourgues 2011, and references therein).

It is tempting to connect the cosmological preference for $N_{\text {eff }}>3$ with the possible additional species from oscillation experiments. In this review, we focus on the physical effects (Section 2), the cosmological observations (Section 3), the possible origin of these effects in terms of particle physics and other explanations (Section 4), and what to expect in the future (Section 5).

Throughout the paper, we use a fiducial cosmology with the best-fit WMAP7 parameters from Komatsu et al. (2011) for illustrations: $\Omega_{\mathrm{b}, 0}=0.0451$ (baryon density today), $\Omega_{c, 0}=$ 0.226 (matter density today), $\Omega_{\Lambda, 0}=0.729$ (dark energy density), $H_{0}=70.3 \mathrm{~km} \mathrm{~s}^{-1} \mathrm{Mpc}^{-1}$ (Hubble parameter today),

\footnotetext{
${ }^{1}$ One extra thermal neutrino specie decoupling at the same time as the standard neutrinos will give $N_{\text {eff }}=4$. Decoupling at earlier times will increase $N_{\text {eff }}$ proportionally to the cube of the temperature ratio.
}

$n_{\mathrm{s}}=0.966$ (index describing the tilt of the power spectrum of primordial density fluctuations $), \Delta_{\mathcal{R}}^{2}\left(k=0.002 \mathrm{Mpc}^{-1}\right)=$ $2.42 \times 10^{-9}$ (amplitude of the primordial power spectrum at $k=0.002 \mathrm{Mpc}^{-1}$ ), and unless otherwise stated $w=-1$ (equation of state of the dark energy). All quoted uncertainties on $N_{\text {eff }}$ are $68 \%$ confidence levels, and all upper limits on $\sum m_{v}$ are $95 \%$.

\section{EARLY UNIVERSE EFFECTS}

In order to understand the observable effects of dark radiation parametrised as $N_{\text {eff }}$, it is illustrative to follow the normal neutrinos during the evolution of the early Universe depicted for the fiducial cosmology in the left column of the timeline in Figure 2:

Neutrino decoupling $\left(T_{v \text { dec }} \approx 1.1 \mathrm{MeV}, \quad z_{v \text { dec }} \approx\right.$ $\left.\mathbf{4 . 5} \times \mathbf{1 0}^{9}\right)$. Neutrinos only interact through the weak interaction and consequently when the early Universe temperature drops below $1 \mathrm{MeV}$, the interaction rate per neutrino drops below the expansion rate, and the neutrinos decouple from the thermal plasma. For neutrino masses below $1 \mathrm{eV}$, the neutrinos are still ultra-relativistic when they decouple and they retain a relativistic Fermi-Dirac phase-space distribution. Their energy distribution is described by a blackbody spectrum with a single temperature, and they may have a non-zero chemical potential (related to lepton asymmetry).

Big bang nucleosynthesis $\left(T_{\text {nuc }} \approx 0.8 \mathrm{MeV}, z_{\text {nuc }} \approx\right.$ $\left.\mathbf{2 . 6} \times \mathbf{1 0}^{9}\right)$. The formation of deuterium (D) and helium (He) nuclei is affected by the neutrinos in two ways. First, electron neutrinos participate in the charged current weak interactions that determine the neutron to proton ratio (e.g. $v_{e}+n \leftrightarrow p+$ $e^{-}$) and, second, they increase the expansion rate as

$$
H^{2}(z) \simeq \frac{8 \pi G}{3}\left(\rho_{\gamma}+\rho_{v}\right),
$$

where $H(z)$ is the Hubble rate and $\rho_{\gamma}$ and $\rho_{\nu}$ are the photon and neutrino densities, respectively. The photon energy density is very well determined from the CMB temperature, so constraints on the expansion in the early Universe, $H(z)$, can be translated directly to $\rho_{v}$ and hence $N_{\text {eff }}$.

Photon re-heating (electron recombination) $\left(T_{\text {rec }} \approx\right.$ $0.2 \mathrm{MeV}, z_{\text {rec }} \approx \mathbf{8 . 5} \times \mathbf{1 0}^{8}$ ). When the electrons and positrons decouple from the thermal plasma, most of them annihilate. The released energy re-heats the photon population, but not the bulk of the neutrinos since they are no longer in thermal equilibrium with the photons. After annihilation

$$
T_{v}=(4 / 11)^{1 / 3} T_{\gamma} .
$$

Because the decoupling of the neutrinos is not instantaneous, some of the high-energy neutrinos are still in thermal equilibrium with the photons and will feel the re-heating, leading to a slight excess of high-energy neutrinos relative to a blackbody spectrum. The extra energy per particle is well 


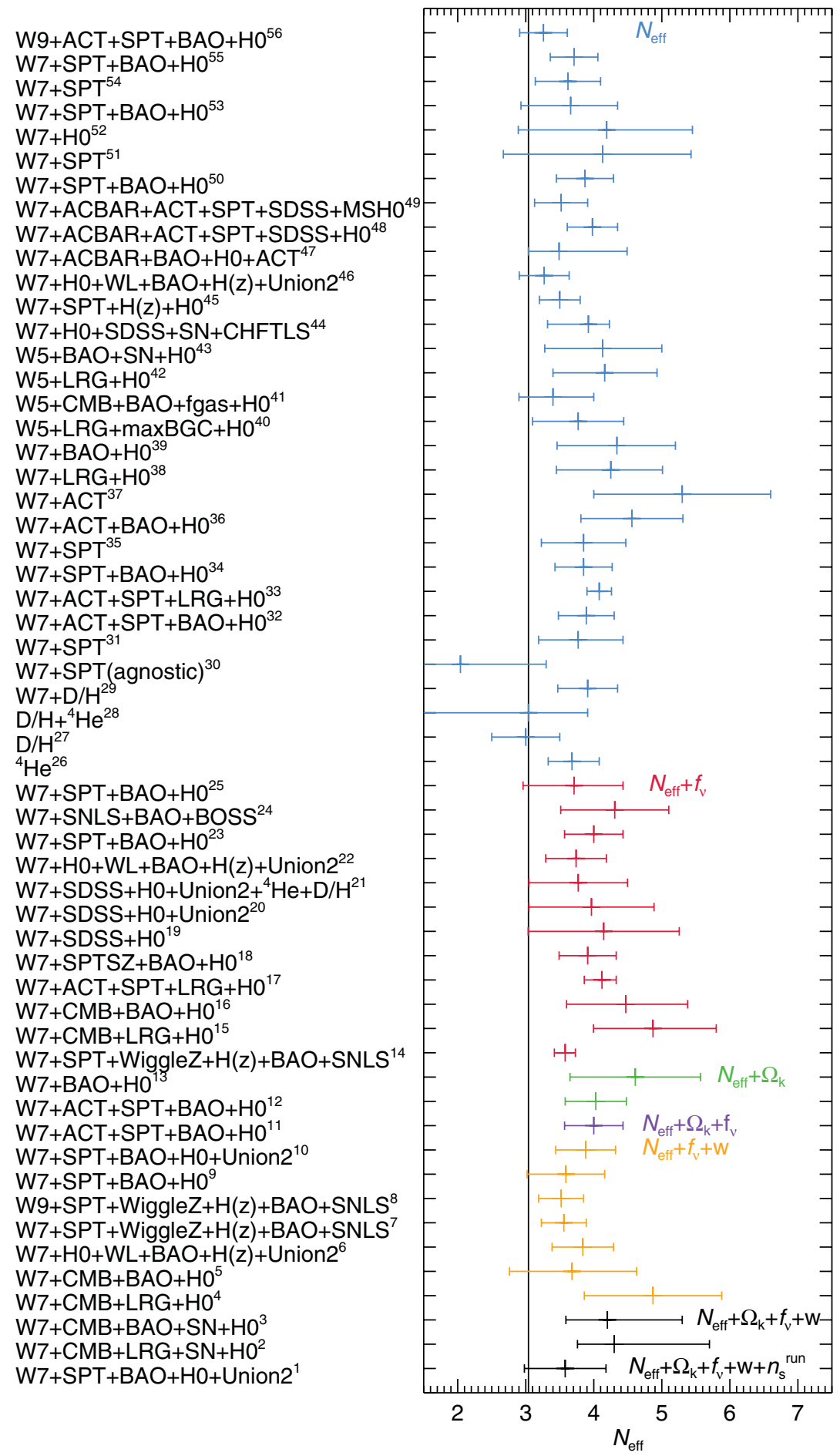

Figure 1. A selection of cosmological $N_{\text {eff }}$ measurements and $68 \%$ confidence intervals from the literature for various combinations of models and data sets. W denotes WMAP followed by data release. The models are all $\Lambda \mathrm{CDM}$ plus the extensions given on the plot. Results are from: 1,9,10,23,50 Joudaki (2012), ${ }^{2,3}$ Gonzalez-Garcia, Maltoni, \& Salvado (2010), , 4,5,13,15,16 Hamann et al. (2010), ${ }^{6,22,46}$ Wang et al. (2012), ${ }^{7,8}$ S. Riemer-Sørensen (unpublished), ${ }^{11,12,32} \mathrm{Smith}$ et al. (2012), ${ }^{14}$ Riemer-Sørensen et al. (2013), ${ }^{17,33}$ Archidiacono, Calabrese, \& Melchiorri (2011), ${ }^{18}$ Benson et al. (2011), ${ }^{19,20,21}$ Giusarma et al. (2011), ${ }^{24}$ Zhao et al. (2012), ${ }^{25,51,52,53}$ Giusarma, de Putter, \& Mena (2012), ${ }^{26}$ Izotov \& Thuan (2010), ${ }^{27}$ Pettini \& Cooke (2012), ${ }^{28}$ Mangano \& Serpico (2011), ${ }^{29}$ Nollett \& Holder (2011), ${ }^{30,31}$ Audren et al. (2012), ${ }^{34,35}$ Keisler et al. (2011), ${ }^{36,37}$ Dunkley et al. (2011), ${ }^{38,39}$ Komatsu et al. (2011), ${ }^{40,42,43}$ Reid et al. (2010), ${ }^{41}$ Mantz, Allen, \& Rapetti (2010), ${ }^{44}$ Xia et al. (2012), ${ }^{45}$ Moresco et al. (2012), ${ }^{47}$ Gonzalez-Morales et al. (2011), ${ }^{48,49}$ Calabrese et al. (2012), ${ }^{54,55} \mathrm{Hou}$ et al. (2012), and ${ }^{56} \mathrm{Hinshaw}$ et al. (2012). 


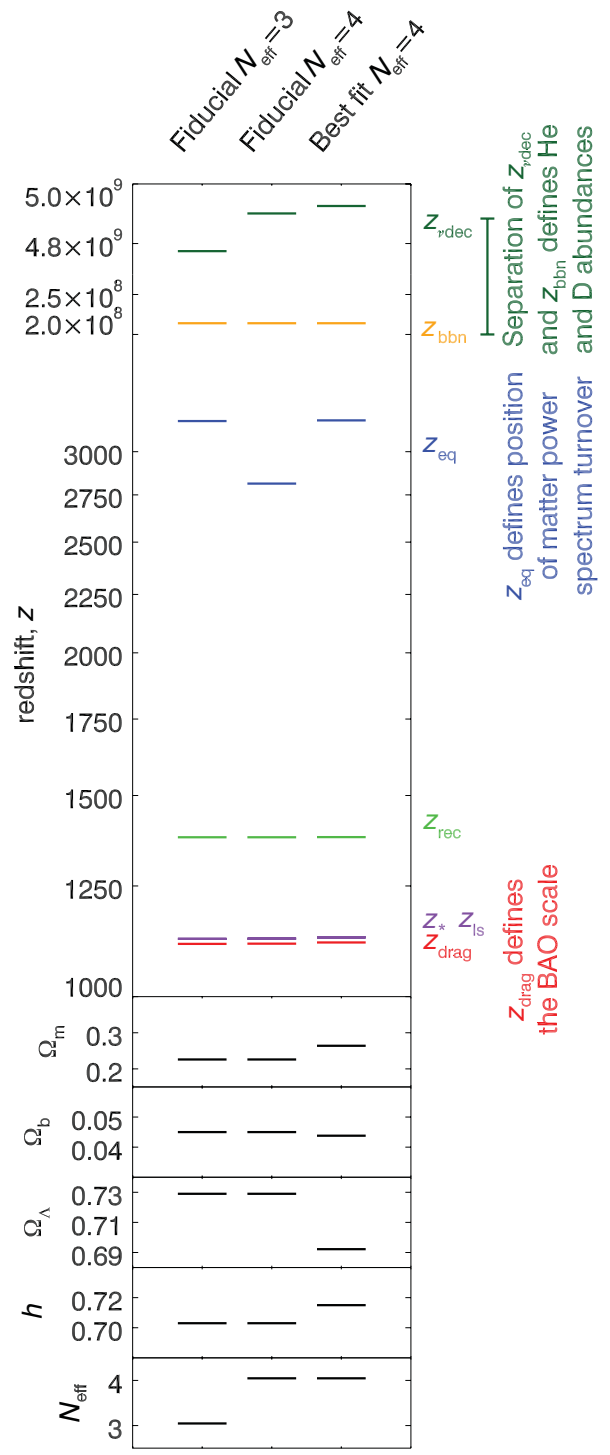

Figure 2. The redshifts of important events in the early Universe (described in the main text) and their dependence on $N_{\text {eff }}$ (the first two columns) and on cosmological parameters for fixed $N_{\text {eff }}=4$ (the second and third columns). Changing $N_{\text {eff }}$ mainly changes the redshift of matter-radiation equality $\left(z_{\text {eq }}\right)$ for fixed cosmological parameters. However, this change can be compensated by increasing the values of $H_{0}$ and $\Omega_{\mathrm{m}, 0}$. Note that the neutrino decoupling remains early allowing one to distinguish between the first and third scenarios through deuterium and helium abundances.

approximated by increasing the number of particles slightly, and is commonly absorbed into the definition of $N_{\text {eff }}$ via

$$
\rho_{v}=N_{\text {eff }} \frac{7 \pi^{2}}{120} T_{v}^{4},
$$

where $\rho_{v}$ refers to the neutrino energy density when they are still relativistic, and the factor $\left(7 \pi^{2} / 120\right) T_{v}^{4}$ is the energy density in one species of relativistic neutrinos. The additional re-heating leads to $N_{\text {eff }}=3.046$ for $N_{v}=3$.

Matter-radiation equality $\left(T_{\mathrm{eq}} \approx 0.8 \mathrm{eV}, z_{\mathrm{eq}} \approx 3200\right)$. Relativistic neutrinos behave like radiation and consequently change the time of radiation-matter equality (Lesgourgues
\& Pastor 2006):

$$
\frac{a_{\mathrm{eq}}}{a_{0}}=\frac{1}{1-f_{v}} \frac{\Omega_{\mathrm{r}}}{\Omega_{\mathrm{m}}},
$$

where $\Omega_{\mathrm{m}}$ is the total matter density, $\Omega_{\mathrm{r}}$ is the radiation density, and $f_{v}=\Omega_{v} / \Omega_{\mathrm{m}}$ is the neutrino fraction. The matterradiation equality determines when the density perturbations can begin to grow and form structures through gravitational collapse. If this is pushed to later times, the peak of the matter power spectrum moves to larger scales (because the particle horizon is larger when the structures begin to collapse).

Recombination makes the Universe neutral $\left(T_{\text {rec }} \approx\right.$ $0.3 \mathrm{eV}, z_{\text {eq }} \approx \mathbf{1 3 7 0}$ ). Once the temperature has dropped sufficiently below the ionisation energy of hydrogen, the electrons will couple to the nuclei and the Universe becomes neutral ( $z_{\text {rec }}$ is here defined as when half the electrons are bound). Since the neutrinos have already decoupled a long time ago, they are unaffected by this, but the recombination is the beginning of the CMB era.

Photon decoupling and last scattering $\left(T_{*} \approx T_{\mathrm{ls}} \approx\right.$ $\left.\mathbf{0 . 2 5} \mathrm{eV}, z_{*} \approx z_{\mathrm{ls}} \approx \mathbf{1 1 0 0}\right)$. When the temperature of the Universe has dropped to $\approx 0.25 \mathrm{eV}$, the collision rate between photons and free electrons drops below the expansion rate and photons propagate freely. The last scattering between photons and electrons is observed today as the CMB. The neutrinos do not care about this, but the CMB is one of the most powerful probes available to study the early Universe (see Section 3.2).

Baryon decoupling and the drag epoch $\left(T_{\text {drag }} \approx\right.$ 0.24-0.25 eV, $z_{\text {drag }} \approx \mathbf{1 0 2 0}-\mathbf{1 0 6 0}$ ). Even after the photonelectron scattering ceases and the mean-free path of photons becomes effectively infinite, a small number of the photons do still interact with the far less numerous baryons ( $n_{\mathrm{b}} / n_{\gamma} \approx 10^{-10}$ ), prolonging the pressure wave in the baryons. This is essentially a difference between when the photons stop noticing the baryons (decoupling), and when the baryons stop noticing the photons. This delay is known as the drag epoch, at the end of which the density fluctuations that form the seeds of structure are frozen in. This event does not affect the neutrinos, but the decoupling allows the baryons to cluster in the dark matter potentials initiating the formation of observable structures.

Becoming non-relativistic $\left(T_{\mathrm{nr}} \approx 0.05 \mathrm{eV}, z_{\mathrm{nr}} \approx 200 \mathrm{de}-\right.$ pending on $\left.\sum \boldsymbol{m}_{v}\right)$. At least two of the neutrino mass eigenstates are massive so their velocities will decay adiabatically with the expansion of the Universe, and eventually they become non-relativistic $\left(m_{v} c^{2}>p_{\nu} c\right)$ (Lesgourgues \& Pastor 2006; de Bernardis et al. 2009)

$$
z_{\mathrm{nr}} \approx 2 \times 10^{3} \frac{m_{v, i}}{\mathrm{eV}}
$$

The last eigenstate is allowed to be massless and if this is the case, it will remain relativistic forever. When the neutrinos become non-relativistic, they behave like a species 

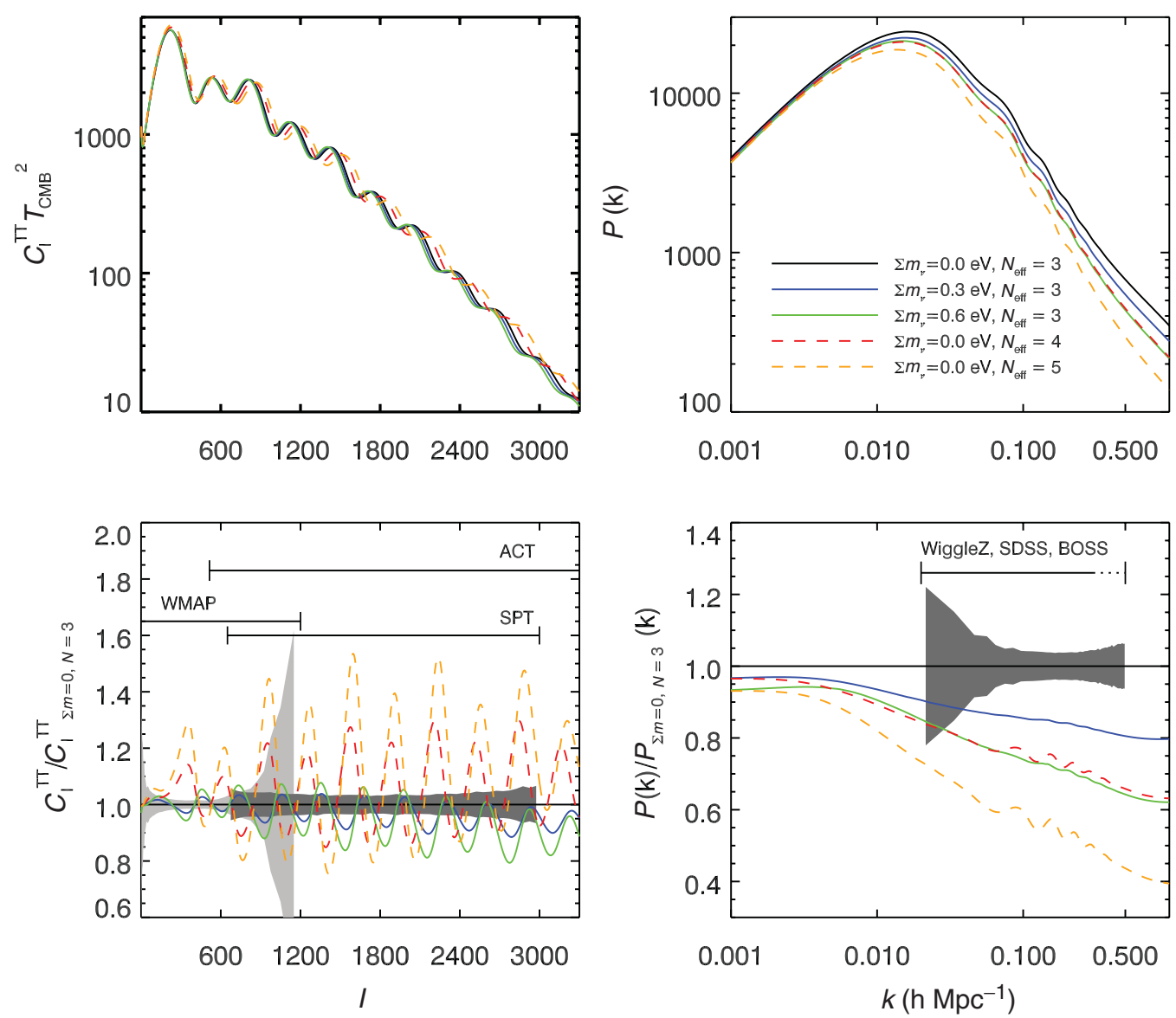

Figure 3. Illustration of the CMB (left) and matter (right) power spectra for a fiducial cosmology and how they change for varying neutrino mass (solid lines, we vary $f_{v}=\Omega_{v} / \Omega_{\mathrm{m}}=\sum m_{v} /\left(93.14 \mathrm{eV} \Omega_{\mathrm{m}} h^{2}\right)$ ), and effective number of neutrinos (dashed lines) fixing all other parameters. The bottom two plots show the ratio of the power spectra when varying $\sum m_{v}$ and $N_{\text {eff }}$ relative to $\Sigma m_{v}=0 \mathrm{eV}, N_{\text {eff }}=0 . \sum m_{\nu}$ does not affect the CMB power spectrum much, but changes the matter power spectrum. The effect of $N_{\text {eff }}$ is clearly visible for small scales (high values of $l$ ) in the CMB power spectrum, and the two parameters are clearly degenerate for the matter power spectrum unless the peak position is very precisely measured. The horizontal lines indicate the coverage by current and near-future experiments, and the shaded areas indicate the relative magnitude of the uncertainties for WMAP (light grey) and SPT (dark grey) in the CMB plot, and WiggleZ (dark grey) for galaxy surveys.

of warm/hot dark matter, slowing the growth of density fluctuations on scales smaller than their free-streaming length ${ }^{2}$ (see Section 3.3).

\section{OBSERVABLE EFFECTS}

The main effect of dark radiation is to alter the expansion rate and the time of matter-radiation equality, which affects nucleosynthesis, the $\mathrm{CMB}$, and structure formation. The relevant observables for nucleosynthesis are the deuterium and helium abundances, for the CMB it is the power spectrum of temperature and polarisation fluctuations expressed in terms of spherical harmonics, and for structure formation it is the (matter) density power spectrum inferred from e.g. galaxy

\footnotetext{
2 The free-streaming length is the average distance the neutrinos propagate freely, and for kinematic reasons neutrinos cannot be confined (or kept out of) regions smaller than the free-streaming length.
}

surveys. Figure 3 illustrates the effects of increasing the neutrino mass (solid lines) and number of species (dashed lines) on the $\mathrm{CMB}$ and density power spectra. In the following sections, we will discuss these observable effects in detail.

What is probed directly by cosmological analyses is not the neutrino mass, but the neutrino density, $\rho_{\nu}$, which can be expressed in terms of the sum of neutrino masses (Lesgourgues \& Pastor 2006)

$$
\Omega_{v}=\frac{\rho_{v}}{\rho_{\mathrm{c}}}=\frac{\sum_{i=1}^{N_{v}} m_{v, i}}{93.14 \mathrm{eV} h^{2}},
$$

where $\rho_{\mathrm{c}}$ is the critical energy density for a flat Universe. Because of the smallness of the measured mass differences relative to the upper limits, it is reasonable to assume that if the individual neutrino masses are near the upper limit, they are effectively equal. If the individual masses are close to the lower limit, the hierarchy will play an important role (Jimenez et al. 2010). 


\subsection{Big bang nucleosynthesis}

The presence of dark radiation mainly alters the nucleosynthesis by increasing the expansion rate, leading to higher deuterium and helium abundances than are observed today (Lesgourgues \& Pastor 2006; Steigman 2012).

\subsubsection{Helium}

The freeze-out of weak interactions determines the neutron/proton ratio, which will eventually determine the helium fraction $\left(Y_{p}=\rho_{\mathrm{He}} / \rho_{\text {baryon }}\right)$, since all available neutrons either become bound in helium or decay. Increasing the expansion rate will induce earlier freeze-out of the weak interaction (see Figure 2), and more neutrons will be available, leading to a higher helium fraction.

Helium-4 $\left({ }^{4} \mathrm{He}\right)$ is the nucleosynthesis relic most sensitive to $N_{\text {eff }}$ but because it is produced in stars it is not trivial to obtain a robust value of the primordial abundance. High quoted values of $N_{\text {eff }}$ from nucleosynthesis are usually driven by the adopted value of $Y_{p}$ and its uncertainty. Since the 1990s the observed value has increased from $Y_{p}=0.2462 \pm$ 0.0015 (Izotov et al. 1999) to $Y_{p}=0.2565 \pm 0.001$ (stat.) \pm 0.005 (syst.) (Izotov \& Thuan 2010), which drives the value of $N_{\text {eff }}$ up.

\subsubsection{Deuterium}

The deuterium abundance is determined by how much deuterium is fused into helium- 4 after deuterium formation has ceased because of too low neutron and proton densities towards the end of the nucleosynthesis. Increasing $N_{\text {eff }}$ will speed up the expansion (but not as much as when helium formed), allowing for less deuterium to be fused into helium and consequently leading to a higher deuterium abundance (Nollett \& Holder 2011).

\subsubsection{Lithium}

The lithium abundance predicted from standard nucleosynthesis is a factor of three higher than that observed in metalpoor halo stars. Increasing $N_{\text {eff }}$ does not change this problem (Steigman 2012).

\subsubsection{Results from nucleosynthesis}

There have been a number of recent attempts to derive $N_{\text {eff }}$ from nucleosynthesis alone (e.g. Mangano \& Serpico 2011; Pettini \& Cooke 2012), which all seem to be consistent with $N_{\text {eff }}=3$. Analyses combining nucleosynthesis and CMB appear to prefer $N>3$ (e.g. Nollett \& Holder 2011; Hamann et al. 2011).

\subsection{Cosmic microwave background}

The distribution of hot and cold spots in the CMB is given by the fluctuations in the photon-baryon plasma at the surface of last scattering. These fluctuations are generated by sound waves in the plasma, where the repulsive electrostatic force is balanced by the gravitational attraction of dark matter po- tentials that have been growing since $z_{\text {eq }}$ (see Figure 2). They manifest in the observed CMB power spectrum as actual oscillations, as shown in Figure 3. An accurate measurement of the positions and heights of the peaks and troughs can give us two important pieces of information about the relativistic energy density.

First, the amplitude of the oscillations relates to the matterto-radiation density ratio. Pressure support of the compression wave in the plasma causes the gravitational potential to decay, enhancing the amplitude of these perturbations. This enhancement occurs only for perturbations that have already crossed the horizon before matter-radiation equality ( $\mathrm{Hu} \&$ Sugiyama 1996), which is true for the third and subsequent peaks. This is why the third peak is enhanced with respect to the second, and the amplitude ratio of the first to third peaks gives a good measurement of $z_{\text {eq }}$, and so $N_{\text {eff }}$.

Second, the peak positions give the size of the sound horizon at decoupling, namely the maximum distance the acoustic oscillations can travel before the sound speed in the fluid drops to zero. This relates directly to the Hubble rate, and so is also influenced by $N_{\text {eff }}$. Notice from Figure 3 that increasing the value of $N_{\text {eff }}$ moves the acoustic peaks to smaller scales (since the sound horizon, $r_{\mathrm{s}}$, becomes smaller for increasing $N_{\text {eff }}$ ), whereas in the matter power spectrum the turnover moves to larger scales (related to the change of $z_{\text {eq }}$ seen in Figure 2). This anti-correlation between effects in the acoustic peaks in the CMB and the turnover in the matter power spectrum provides an important consistency check between the early- (pre-CMB) and late-time (post-CMB) physics.

In addition, we must consider the damping of the $\mathrm{CMB}$ power spectrum 'tail' on small scales. Photons in the photonbaryon fluid will diffuse out of overdense regions and generate an anisotropic stress that damps the oscillations on small scales $(\ell>200)$. This effect is called Silk or diffusion damping. The neutrinos contribute to the small-scale damping both indirectly - by changing the expansion and thereby the damping from the photons - and directly - through their own free streaming.

Increasing the expansion rate by adding extra relativistic material will increase the Silk damping because structures have less time to grow and are consequently easier to wipe out through diffusion. Similarly, increasing the expansion rate increases the helium abundance, which also increases the damping because the helium abundance controls the number of free electrons per unit baryon mass.

Neutrinos contribute to the damping through anisotropic stress and free-streaming effects (Hu et al. 1995). Similar to the photons, the neutrinos have a viscosity that creates an anisotropic stress and consequently generates damping on small scales. Fluctuations in the neutrino density lead to a shift in the positions of the acoustic peaks. These effects are non-negligible, as at the time of decoupling, the standard neutrinos contribute around $10 \%$ of the total energy density of the Universe. Because the neutrinos are free streaming, they suppress structure formation on scales smaller than their free-streaming length (see Section 3.3). The combination of 
these effects tells us something more than simply the density of the relativistic species, and can provide details on both the mass and viscosity of the dark radiation.

\subsubsection{Results from $C M B$}

CMB observations of the first three acoustic peaks in the temperature power spectra from WMAP have accurately measured the redshift of matter-radiation equality to be $z_{\text {eq }}=$ $3145_{-139}^{+140}$ (Komatsu et al. 2009), for models where $N_{\text {eff }}$ is allowed to vary. However, WMAP cannot measure the tail of the CMB temperature power spectrum $(\ell>1200)$, and so is unable to put an upper limit on $N_{\text {eff }}$, giving only a lower limit of $N_{\text {eff }}>2.7$ (95\% confidence level). An upper limit can be found either by combining WMAP with late-time measurements of the matter density (SN, BAO, or galaxy power spectra), or through measurements of the CMB tail. When combined with small-scale CMB experiments such as the ACT or South Pole Telescope (SPT), the constraints improve (see Figure 1), giving $N_{\text {eff }}=5.3 \pm 1.3$ from $W M A P+$ ACT (Dunkley et al. 2011) and $N_{\text {eff }}=3.85 \pm 0.62$ from WMAP + SPT (Keisler et al. 2011).

In terms of the neutrino mass, the CMB alone cannot provide very strong constraints. In order to have a measurable effect on the CMB, the neutrinos would have to be nonrelativistic before photon decoupling, but current cosmological limits indicate that they were relativistic at this time. The upper limit imposed by WMAP is $\sum m_{v}<1.3 \mathrm{eV}$ (Komatsu et al. 2009), which is close to (in fact beyond!) the absolute limit of $\sum m_{v}<1.5 \mathrm{eV}$ one can theoretically expect to derive from CMB alone (Ichikawa, Fukugita, \& Kawasaki 2005). However, the CMB does play an important role of providing good 'base-level' constraints on the matter density $\left(\Omega_{\mathrm{m}}\right)$ and the amplitude of primordial fluctuations $\left(\Delta_{\mathcal{R}}^{2}\right)$, which need to be jointly measured when combining the CMB with other cosmological data sets to measure the neutrino mass.

\subsection{Large-scale structure}

Like the CMB, the large-scale structure is affected by neutrinos through the expansion and their free-streaming effects. The expansion determines the time available for sound waves to propagate in the baryon-photon fluid before recombination, which will imprint on the BAO scale measurable from both the CMB and in the galaxy power spectrum (see Figure 2). Increasing $N_{\text {eff }}$ pushes the BAO peak towards smaller scales, indicated by a slight shift of the positions of the 'wiggles' in the galaxy power spectrum in Figure 3. The position of the matter power spectrum turnover is determined by the time available for structures to form, and is consequently pushed to smaller scales as matter-radiation equality moves to later times with increasing $N_{\text {eff }}$. However, this effect is degenerate with effects from the ordering of the neutrino masses (the hierarchy) that dictates whether one-third or two-thirds of the neutrinos will be 'heavy' or 'light'. The light ones will be radiation-like for longer and enhance structure growth on large scales (Jimenez et al. 2010; Wagner, Verde, \& Jimenez 2012).

Massive neutrinos (or other particles) will also slow down structure formation due to their free-streaming properties. After decoupling, their masses will lead to an adiabatic decay of their thermal velocities as (Komatsu et al. 2011)

$$
v_{\text {thermal }}=151(1+z)\left(1 \mathrm{eV} / m_{v}\right) \mathrm{km} \mathrm{s}^{-1} \text {. }
$$

Eventually, they become non-relativistic and behave as a species of warm/hot dark matter, suppressing the growth of density fluctuations on scales characterised by their free-streaming length at the time when they become nonrelativistic (Lesgourgues \& Pastor 2006)

$$
\begin{aligned}
k_{\mathrm{FS}} & =\sqrt{\frac{3}{2}} \frac{H(t)}{v_{\text {thermal }}(t)(1+z)} \\
& \approx 0.82 \frac{\sqrt{\Omega_{\Lambda}+\Omega_{m}(1+z)^{3}}}{(1+z)^{2}}\left(\frac{m_{v}}{1 \mathrm{eV}}\right) h \mathrm{Mpc}^{-1} .
\end{aligned}
$$

This happens because the neutrinos cannot cluster on scales smaller than their free-streaming length and consequently the density fluctuations are suppressed by a constant factor proportional to the neutrino density, and will grow more slowly than without the presence of massive neutrinos. The effect is observable in the density power spectrum as a suppression of structure on small scales shown in the right part of Figure 3. However, the presence of neutrinos also enhances the density perturbations on large scales (by adding to the radiation density for fixed $\Omega_{\mathrm{m}, 0}$ ), which boosts structure formation in general (Hou et al. 2011).

The evolution of the density perturbations can be predicted by solving the coupled set of Einstein and Boltzman equations. For small density perturbations, the linearised equations are sufficient (calculated numerically e.g. using $\mathrm{CAMB}^{3}$ or $\mathrm{CLASS}^{4}$ ), but when the density perturbations grow sufficiently large, the higher order, non-linear terms become important and have to be included, which is computationally very intensive (and not yet solved analytically). Instead, one has to rely on second-order perturbation theory (e.g. Saito, Takada, \& Taruya 2009; Taruya et al. 2012) or simulations (e.g. Smith et al. 2003; Jennings, Baugh, \& Pascoli 2010). Numerical solutions show that for $f_{v}=\Omega_{v} / \Omega_{\mathrm{m}}<0.07$, the suppression is $\delta P / P=-8 f_{v}$ for linear structure formation (Hu, Eisenstein, \& Tegmark 1998) and the effect increases for non-linear structure formation ( $8 \rightarrow 9.6$; Brandbyge et al. 2008; Brandbyge \& Hannestad 2009, 2010; Viel, Haehnelt, \& Springel 2010; Agarwal \& Feldman 2011).

The neutrinos affect the total matter distribution which is dominated by the dark matter, and consequently not directly observable. Instead, we use tracers such as galaxies or the absorption lines from neutral hydrogen clouds along the line

\footnotetext{
${ }^{3}$ http://camb.info

${ }^{4}$ http://lesgourg.web.cern.ch/lesgourg/class.php
} 

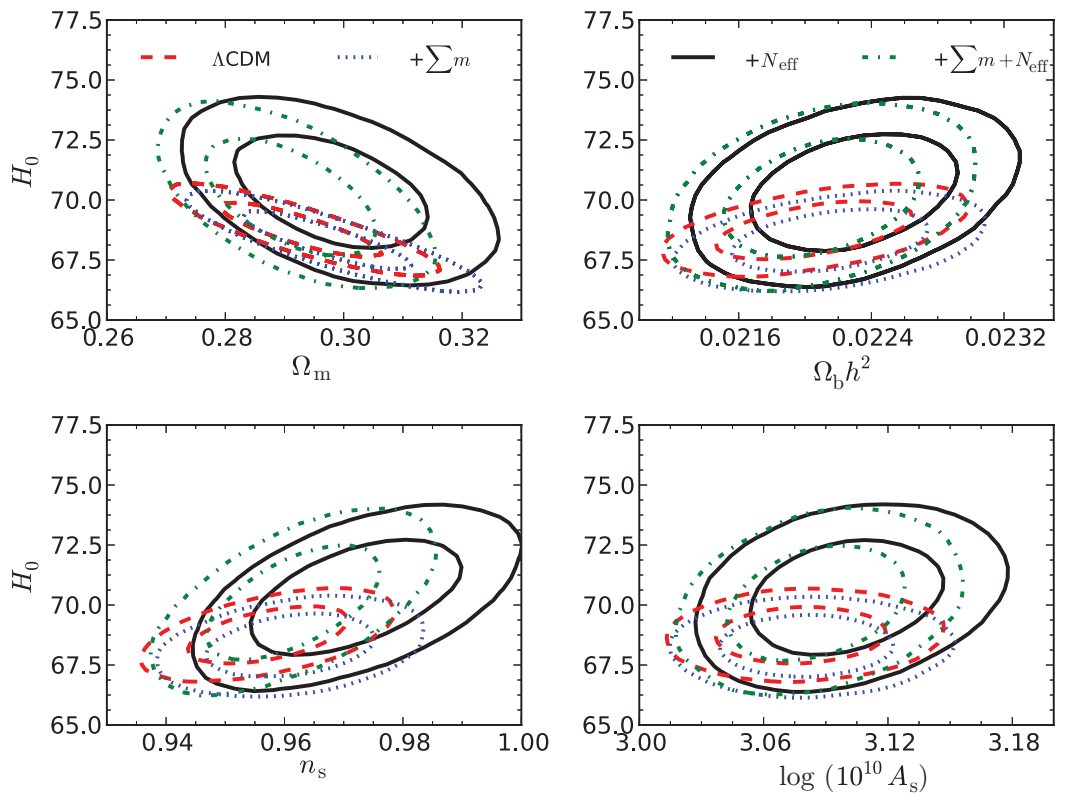

Figure 4. The $68 \%$ and $95 \%$ confidence limit contours for fitting CMB $(W M A P 7+\mathrm{SPT})+$ large-scale structure (WiggleZ) $+H(z)+\mathrm{BAO}$ (SDSS, 6dFGS, BOSS) $+\mathrm{SN}$ (SNLS) data with $\Lambda \mathrm{CDM}$ (dashed red) and extensions to the model with $\Sigma m_{v}$ (dashed blue), $N_{\text {eff }}$ (dot-dashed green), and both $\Sigma m_{v}$ and $N_{\text {eff }}$ (solid black) from Riemer-Sørensen et al. (2013). The resulting contours are consistent with each other for all parameters, clearly showing that the preference for high values of $N_{\text {eff }}$ is not related to an offset in the measurement of a single standard $\Lambda$ CDM parameter, but can be added within the standard parameter uncertainties.

of sight to distant quasars (Lyman alpha forest), for which the power spectrum may differ slightly from the total matter power spectrum if baryons do not perfectly trace the dark matter. Depending on the tracer, the bias, $b$, defined as $P_{\text {obs }}=$ $b^{2} P_{\mathrm{m}}$, might be scale dependent or scale independent (Schulz $\&$ White 2006). A scale-dependent bias can introduce a degeneracy with the slope change induced by the neutrinos.

For a more in-depth, technical account of massive neutrinos in cosmology, see Lesgourgues \& Pastor (2006) or Wong (2011).

\subsubsection{Results from large-scale structure}

Large-scale structure data alone cannot constrain all the cosmological parameters, so they are usually analysed jointly with CMB data. Currently, there is no precise measurement of the matter power spectrum turnover (Poole et al., 2013), so large-scale structure data mainly improve on the $\sum m_{v}$ constraints, and are consequently most important when fitting for $\sum m_{v}$ and $N_{\text {eff }}$ simultaneously rather than for $N_{\text {eff }}$ alone. The current limits of $\sum m_{v}<0.3 \mathrm{eV}$ for $\Lambda \mathrm{CDM}+$ $\sum m_{v}$ (Moresco et al. 2012; Xia et al. 2012; de Putter et al. 2012; Riemer-Sørensen et al. 2012) and $\sum m_{v}<0.65 \mathrm{eV}$ for $\Lambda \mathrm{CDM}+\sum m_{v}+N_{\text {eff }}$ (e.g. Hamann et al. 2010; Joudaki 2012; Riemer-Sørensen et al. 2013; Wang et al. 2012) are stronger than what is provided by particle physics experiments.

\section{DISCUSSION}

\subsection{Do we need $N_{\text {eff }}$ ?}

Perfect measurements of the standard $\Lambda$ CDM parameters do not exist. Adding $N_{\text {eff }}$ to the cosmological model does not shift the best-fit values of the standard $\Lambda \mathrm{CDM}$ parameters in any systematic way, indicating that the preference for high $N_{\text {eff }}$ is not due to an offset measurement of a single parameter, but can be accommodated within the uncertainties of the standard parameter values.

Figure 4 compares the contours from fitting a range of cosmological data (see caption) with a standard $\Lambda$ CDM model alone, and with $\sum m_{v}$ and $N_{\text {eff }}$ extensions individually and combined. Naturally the constraints are tighter for pure $\Lambda \mathrm{CDM}$, since there are fewer parameters to constrain, but overall the constraints are consistent for all parameters. Adding $\sum m_{v}$ to the model (dotted blue) does not change the $\Lambda \mathrm{CDM}$ parameters or uncertainties significantly, whereas adding $N_{\text {eff }}$ (dot-dashed green) increases the values and uncertainties, particularly for $H_{0}$. Adding both parameters simultaneously (solid black) increases the preferred values of $H_{0}$ and $n_{\mathrm{s}}$ but also the uncertainties, so the values remain consistent with the pure $\Lambda \mathrm{CDM}$ case. The shift in $n_{\mathrm{s}}$ happens because the increased expansion $\left(H_{0}\right)$ changes the height of the peaks in the $\mathrm{CMB}$, as it corresponds to increasing the physical matter density. Increasing $N_{\text {eff }}$ recovers the details 
of the CMB peaks (because the original ratio of matter to radiation is recovered, restoring the details of the Silk damping), but with too much power on large scales, which can be eliminated shifting the primordial power spectrum from very red $\left(n_{\mathrm{s}}=0.96\right)$ to slightly less red $\left(n_{\mathrm{s}}=0.98-1.0\right)$.

\subsection{Priors}

Since most constraints on $N_{\text {eff }}$ are obtained through Bayesian analyses, one could be concerned that the preference for $N_{\text {eff }}>3$ originates in the choice of priors on the model parameters. Both Gonzalez-Morales et al. (2011) and Hamann (2012) investigated this and found no significant shifts in the $N_{\text {eff }}$ constraints for various selections of priors.

\subsection{Beyond $\Lambda \mathrm{CDM}+\sum m_{v}+N_{\text {eff }}$}

Other physical effects such as curvature, varying equation of state, running of the spectral index, etc., could mimic $N_{\text {eff }}>3$ if not properly accounted for in the modelling. Joudaki (2012) demonstrated that the deviation from $N_{\text {eff }}=3.046$ is diminished if allowing for curvature, varying equation of state, running of the spectral index, and/or the helium fraction by fitting to a combination of $\mathrm{CMB}+\mathrm{BAO}+H S T+\mathrm{SN}$ data. However, for all parameter combinations, the preferred value of $N_{\text {eff }}$ was still above three (see Figure 1), and only when more than one extra parameter extension of the $\Lambda \mathrm{CDM}$ cosmology (e.g. curvature and varying equation of state in addition to $N_{\text {eff }}$ and $\sum m_{v}$ ) were considered, the preferred value of $N_{\text {eff }}$ became consistent with three within one standard deviation. Consequently the preference for $\mathrm{N}_{\text {eff }} \neq 3$ does not appear to be strongly dependent on the complexity of the cosmological model. A similar conclusion was reached by Menegoni et al. (2012), who concluded that even when varying both the helium fraction and the fine structure constant on top of $\Lambda \mathrm{CDM}+N_{\text {eff }}$, the preferred value of $N_{\text {eff }}$ remained high.

Audren et al. (2012) took an alternative approach and separated cosmological effects originating directly from early Universe conditions from those related to later time effects, when analysing CMB data. Marginalising over the late time effects, they found the preferred value of $N_{\text {eff }}$ to be consistent with three, and consequently suggested that the preference for high values of $N_{\text {eff }}$ does not relate to changed expansion in the early Universe, but rather to our understanding and modelling of late time effects.

\subsection{Thermalisation}

All current analyses relating the dark radiation to neutrinos rely on an assumption of the sterile neutrinos being in thermal equilibrium with the photons before the neutrinos decouple. However, this is an ad hoc assumption and a full thermalisation calculation involving momentum-dependent density matrices for three active and $\Delta N$ sterile flavours has never been performed (Abazajian et al. 2012).
The thermalisation can be suppressed in the presence of large lepton asymmetry (more leptons than anti-leptons; Foot \& Volkas 1995). In that case, the mass constraints of $\sum m_{v}<$ $0.3 \mathrm{eV}$ from large-scale structure do not apply because they rely on the assumption of thermalisation (Hannestad, Tamborra, \& Tram 2012; Mirizzi et al. 2012). This allows for a joint interpretation of the cosmology and particle physics results under very specific early Universe conditions. The necessary lepton asymmetry $\left(L=\left(n_{f}-n_{\bar{f}}\right) / n_{\gamma} \approx 10^{-2}\right)$ is below any observational constraints, but well above the usually assumed value which is of the same order of magnitude as the baryon asymmetry $\left(L \approx 10^{-10}\right)$. Large neutrino asymmetries can be generated by the active-sterile neutrino mixing without the need to invoke new physics (Foot, Thomson, \& Volkas 1996).

\subsection{Non-standard decoupling}

The number of relativistic degrees of freedom depends on the decoupling processes involved. In the three-neutrino framework, the non-instantaneous decoupling of the standard model neutrinos leads to an expected value of $N_{\text {eff }}=$ 3.046 for standard decoupling. However, non-standard decoupling can maximally increase this value to $N_{\text {eff }}=3.12$ for three neutrino species (Mangano et al. 2006), and consequently non-standard decoupling cannot alone explain the preference for the currently observed high values of $N_{\text {eff }}$.

\subsection{Particle properties}

The value of $N_{\text {eff }}>3$ is often interpreted as an extra species of a non-interacting relativistic neutrino-like particle. If this is the case, it should behave as a neutrino during the evolution of density perturbations which is can be characterised by a relation between its effective sound speed and viscosity parameter of $c_{\mathrm{eff}}^{2}=c_{\mathrm{vis}}^{2}=1 / 3$ (Smith et al. 2012). Here, $c_{\text {vis }}$ describes the ability of the particles to free-stream out of gravitational potentials, and $c_{\text {eff }} \neq 1 / 3$ allows for the existence non-adiabatic pressure perturbations. If the dark radiation is composed of a particle with significant interaction, these parameters can take other values (Basbøll et al. 2009), thus allowing us to distinguish between hypothesised species (Trotta \& Melchiorri 2005). This was tested by Archidiacono et al. (2011) using a combination of CMB + large-scale structure $+H S T$ data. They found $N_{\text {eff }}>3$ at $2 \sigma\left(N_{\text {eff }}=4.15_{-0.23}^{+0.19}\right)$ and $c_{\text {eff }}^{2}=0.24_{-0.02}^{+0.03}$ consistent with $1 / 3$ while $c_{\mathrm{vis}}^{2}$ remains unconstrained. This result is consistent with a relativistic non-interacting particle but is not a unique signature of such a particle.

For the specific case of hypothetical sterile neutrinos there is another effect to consider, which could change the nucleosynthesis, namely the distortion of the electron-neutrino phase space distribution through neutrino oscillations. For the parameters favoured by short baseline experiments, this effect is negligible because the sterile and active neutrinos will be in thermal equilibrium and consequently share the same phase space distribution. 


\subsection{Time evolution of $N_{\text {eff }}$}

There have been a number of attempts to derive $N_{\text {eff }}$ from nucleosynthesis alone (e.g. Mangano \& Serpico 2011; Pettini \& Cooke 2012) that all seem to be consistent with $N_{\text {eff }}$ $=3$, while analyses combining nucleosynthesis and $\mathrm{CMB}$ seem to prefer $N>3$ (e.g. Nollett \& Holder 2011; Hamann et al. 2011). It is concerning that the preference for $N_{\text {eff }}>3$ is present in all analyses including CMB, but not preferred by nucleosynthesis alone. This could indicate a systematic error in one of the data sets. However, nucleosynthesis alone relies on very few and notoriously difficult measurements of the deuterium and helium fractions.

The difference between the results could also be interpreted as potential tension between $N_{\text {eff }}$ measured at two different epochs (nucleosynthesis and recombination), where we naively would expect $N_{\text {eff }}$ to be the same. A temporal variation could be explained theoretically by particles decaying to neutrinos and thereby altering their temperature distributions (Ichikawa et al. 2007; Fischler \& Meyers 2011; Eggers Bjaelde, Das, \& Moss 2012; Hooper, Queiroz, \& Gnedin 2012; Menestrina \& Scherrer 2012; Hasenkamp \& Jörn 2012), or heavier particles in thermal equilibrium with the neutrinos, but not the photons (Boehm et al. 2012). Currently there is no experimental evidence for the existence of such particles.

\subsection{Link between particle physics and cosmology}

Is the cosmological dark radiation related to the sterile neutrinos from the oscillation experiments?

The neutrino oscillation results favour a mass of the order of $1 \mathrm{eV}$, which is incompatible with cosmological mass constraints from combinations of CMB and large-scale structure measurements (e.g. $\sum m_{v}<0.3-0.6 \mathrm{eV}$; Riemer-Sørensen et al. 2012). Archidiacono et al. (2012) analysed the short baseline oscillation data together with cosmological data and found that short baseline data and CMB are compatible with both $3+1$ and $3+2$ scenarios, but when adding large-scale structure both scenarios are under serious pressure. However, it has been shown that factors such as initial lepton asymmetry (Section 4.4) can alleviate these constraints by introducing a non-thermal neutrino spectrum for which the neutrino mass constraints from large-scale structure can be avoided (Hannestad et al. 2012; Mirizzi et al. 2012).

\section{FUTURE MEASUREMENTS OF $\boldsymbol{N}_{\text {eff }}$}

Due to its high spatial resolution and several spectral bands, the Planck microwave observatory is expected to measure $N_{\text {eff }}$ with a $1 \sigma$ uncertainty of $0.20-0.25$ (Bashinsky \& Seljak 2004; Hannestad, Tu, \& Wong 2006; G. B. Poole et al., in preparation). The neutrino anisotropy will also lead to a polarisation of the CMB detectable in future experiments. Combining Planck with future small-scale polarisation experiments, a sensitivity of 0.05 may be achievable (Galli et al. 2010; The COrE Collaboration et al. 2011), which would al- most allow for a measurement of the thermal distortion of the neutrino spectrum (the 0.046).

Measuring the position of the peak of the matter power spectrum (the turnover) would give another handle on $N_{\text {eff }}$. G. B. Poole et al. (in preparation) predict that a Euclid-like galaxy survey will be able to constrain $N_{\text {eff }}$ with a precision of $20 \%$ independently of the CMB. However, the position of the turnover can be degenerate with neutrino hierarchy effects (Wagner et al. 2012). The degeneracies between $\sum m_{v}, N_{\text {eff }}$, and the hierarchy allows for a measurement of either of them if the remaining parameters can be 'fixed' by independent data.

Any neutrino-like behaving particle (including sterile neutrinos and axions etc., which decouple early as relativistic and become non-relativistic) can mimic the effect of the neutrinos. If the value of $N_{\text {eff }}>3$ is due to a particle, the result points towards physics beyond the Standard Model, and its existence will have to be confirmed by particle physics laboratory experiments. If no compatible particles are found in laboratory experiments, the cosmological preference for additional species indicates a lack of understanding and modelling of cosmological data, both of which are interesting scenarios.

\section{CONCLUSIONS}

Cosmological data seem to favour the presence of dark radiation at the time of photon decoupling at a $2 \sigma$ level, while short baseline particle physics experiments are best explained by the existence of 1-2 sterile neutrinos. Unfortunately, the details and required particle properties of the two scenarios are hard to reconcile and appear to have separate solutions. No matter whether the cosmological trend is due to new particles or systematics, it is a clear indication of something we do not understand.

\section{ACKNOWLEDGMENTS}

We would like to thank Chris Blake and Julien Lesgourgues for constructive discussions and the anonymous referee for useful comments adding quality to the review.

\section{REFERENCES}

Aad, G., et al. (ATLAS Collaboration) 2012, Phys. Lett. B, 716, 1 Abazajian, K. N., et al. 2012, arXiv:1204.5379

Abe, K., et al. 2011, PhRvL, 107, 041801

Agarwal, S., \& Feldman, H. A. 2011, MNRAS, 410, 1647

Archidiacono, M., Calabrese, E., \& Melchiorri, A. 2011, PhRvD, 84,123008

Archidiacono, M., Fornengo, N., Giunti, C., \& Melchiorri, A. 2012, PhRvD, 86, 065028

Aseev, V. N., et al. 2011, PhRvD, 84, 112003

Audren, B., Lesgourgues, J., Benabed, K., \& Prunet, S. 2013, J. Cosmol. Astropart. Phys. 2, 1

Auger, M., et al. 2012, PhRvL, 109, 032505

Basbøll, A., Bjaelde, O. E., Hannestad, S., \& Raffelt, G. G. 2009, PhRvD, 79, 043512 
Bashinsky, S., \& Seljak, U. 2004, PhRvD, 69, 083002

Benson, B. A., et al. 2013, APJ, 763, 147

Beringer, J., et al. 2012, PhRvD, 86, 010001

Boehm, C., Dolan, M. J., \& McCabe, C. 2012, JCAP, 12, 27

Brandbyge, J., \& Hannestad, S. 2009, JCAP, 5, 2

Brandbyge, J., \& Hannestad, S. 2010, JCAP, 1, 21

Brandbyge, J., Hannestad, S., Haugbølle, T., \& Thomsen, B. 2008, JCAP, 8, 20

Calabrese, E., Archidiacono, M., Melchiorri, A., \& Ratra, B. 2012, PhRvD, 86, 043520

Calabrese, E., Huterer, D., Linder, E. V., Melchiorri, A., \& Pagano, L. 2011, PhRvD, 83, 123504

Cleveland, B. T., Daily, T., Davis, R. Jr, Distel, J. R., Lande, K., Lee, C. K., Wildenhain, P. S., \& Ullman, J. 1998, ApJ, 496, 505

Chatrchyan, S., et al. (CMS Collaboration) 2012, Phys. Lett. B, 716, 30

de Bernardis, F., Kitching, T. D., Heavens, A., \& Melchiorri, A. 2009, PhRvD, 80, 123509

de Putter, R., et al. 2012, ApJ, 761, 12

Dunkley, J., et al. 2011, ApJ, 739, 52

Eggers Bjaelde, O., Das, S., \& Moss, A. 2012, JCAP, 10, 17

Erken, O., Sikivie, P., Tam, H., \& Yang, Q. 2012, PhRvL, 108, 061304

Fischler, W., \& Meyers, J. 2011, PhRvD, 83, 063520

Flambaum, V. V., \& Shuryak, E. V. 2006, EL, 74, 813

Foot, R., Thomson, M. J., \& Volkas, R. R. 1996, PhRvD, 53, 5349

Foot, R., \& Volkas, R. R. 1995, PhRvL, 75, 4350

Fukuda, Y., et al. 1998, PhRvL, 81, 1562

Gagnon, J.-S., \& Lesgourgues, J. 2011, JCAP, 9, 26

Galli, S., Martinelli, M., Melchiorri, A., Pagano, L., Sherwin, B. D., \& Spergel, D. N. 2010, PhRvD, 82, 123504

Giunti, C., \& Laveder, M. 2011, PhRvD, 84, 093006

Giusarma, E., Corsi, M., Archidiacono, M., de Putter, R., Melchiorri, A., Mena, O., \& Pandolfi, S. 2011, PhRvD, 83, 115023

Giusarma, E., de Putter, R., \& Mena, O. 2012, arXiv:1211.2154

Gonzalez-Garcia, M. C., Maltoni, M., \& Salvado, J. 2010, J. High Energy Phys., 4, 56

Gonzalez-Morales, A. X., Poltis, R., Sherwin, B. D., \& Verde, L. 2011, arXiv:1106.5052

Hamann, J. 2012, JCAP, 3, 21

Hamann, J., Hannestad, S., Raffelt, G. G., Tamborra, I., \& Wong, Y. Y. Y. 2010, PhRvL, 105, 181301

Hamann, J., Hannestad, S., Raffelt, G. G., \& Wong, Y. Y. Y. 2011, JCAP, 9, 34

Hannestad, S., Tamborra, I., \& Tram, T. 2012, JCAP, 7, 25

Hannestad, S., Tu, H., \& Wong, Y. Y. 2006, JCAP, 6, 25

Hasenkamp, J., \& Jörn, K. 2012, arXiv:1212.4160

Hinshaw, G., et al. 2012, arXiv:1212.5226

Hooper, D., Queiroz, F. S., \& Gnedin, N. Y. 2012, PhRvD, 85, 063513

Hou, Z., Keisler, R., Knox, L., Millea, M., \& Reichardt, C. 2011, arXiv:1104.2333

Hou, Z., et al. 2012, arXiv:1212.6267

Hu, W., Eisenstein, D. J., \& Tegmark, M. 1998, PhRvL, 80, 5255

Hu, W., Scott, D., Sugiyama, N., \& White, M. 1995, PhRvD, 52, 5498

Hu, W., \& Sugiyama, N. 1996, ApJ, 471, 542

Huber, P. 2011, PhRvC, 84, 024617
Ichikawa, K., Fukugita, M., \& Kawasaki, M. 2005, PhRvD, 71, 043001

Ichikawa, K., Kawasaki, M., Nakayama, K., Senami, M., \& Takahashi, F. 2007, JCAP, 5, 8

Izotov, Y. I., Chaffee, F. H., Foltz, C. B., Green, R. F., Guseva, N. G., \& Thuan, T. X. 1999, ApJ, 527, 757

Izotov, Y. I., \& Thuan, T. X. 2010, ApL, 710, L67

Jennings, E., Baugh, C. M., \& Pascoli, S. 2010, MNRAS, 410, 2081

Jimenez, R., Kitching, T., Peña-Garay, C., \& Verde, L. 2010, JCAP, 5,35

Joudaki, S. 2012, arXiv:1202.0005

Keisler, R., et al. 2011, ApJ, 743, 28

Klapdor-Kleingrothaus, H. V., \& Krivosheina, I. V. 2006, Mod. Phys. Lett., A21, 1547

Komatsu, E., et al. 2009, ApJS, 180, 330

Komatsu, E., et al. 2011, ApJS, 192, 18

Kopp, J., Maltoni, M., \& Schwetz, T. 2011, PhRvL, 107, 091801

Kraus, C., et al. 2005, Euro. Phys. J. C, 40, 447

Lesgourgues, J., \& Pastor, S. 2006, PhR, 429, 307

Mangano, G., Miele, G., Pastor, S., Pinto, T., Pisanti, O., \& Serpico, P. D. 2006, Nuclear Phys. B, 756, 100

Mangano, G., \& Serpico, P. D. 2011, Phys. Lett. B, 701, 296

Mantz, A., Allen, S. W., \& Rapetti, D. 2010, MNRAS, 406, 1805

Melchiorri, A., Mena, O., \& Slosar, A. 2007, PhRvD, 76, 041303

Menegoni, E., Archidiacono, M., Calabrese, E., Galli, S., Martins, C. J. A. P., \& Melchiorri, A. 2012, PhRvD, 85, 107301

Menestrina, J. L., \& Scherrer, R. J. 2012, PhRvD, 85, 047301

Mention, G., Fechner, M., Lasserre, T., Mueller, T. A., Lhuillier, D., Cribier, M., \& Letourneau, A. 2011, PhRvD, 83, 073006

Mirizzi, A., Saviano, N., Miele, G. \& Serpico, P.D. 2012, Phys. Rev. D, 86, 053009 [arXiv:1206.1046 [hep-ph]]

Moresco, M., Verde, L., Pozzetti, L., Jimenez, R., \& Cimatti, A. 2012, JCAP, 7, 53

Nakayama, K., Takahashi, F., \& Yanagida, T. T. 2011, Phys. Lett. B, 697, 275

Nollett, K. M., \& Holder, G. P. 2011, arXiv:1112.2683

Pettini, M., \& Cooke, R. 2012, MNRAS, 425, 2477

Poole, G.B., et al. 2013, MNRAS, 429, 1902

Reid, B. A., Verde, L., Jimenez, R., \& Mena, O. 2010, JCAP, 1, 3

Riemer-Sørensen, S., Parkinson, D., Davis, T. M., \& Blake, C. 2013, ApJ, 763, 89

Riemer-Sørensen, S., et al. 2012, PhRvD, 85, 081101

Saito, S., Takada, M., \& Taruya, A. 2009, PhRvD, 80, 083528

Schulz, A. E., \& White, M. 2006, APh, 25, 172

Smith, A., Archidiacono, M., Cooray, A., De Bernardis, F., Melchiorri, A., \& Smidt, J. 2012, PhRvD, 85, 123521

Smith, T. L., Pierpaoli, E., \& Kamionkowski, M. 2006, PhRvL, 97, 021301

Smith, R. E., et al. 2003, MNRAS, 341, 1311

Steigman, G. 2012, arXiv:1208.0032

Taruya, A., Bernardeau, F., Nishimichi, T., \& Codis, S. 2012, PhRvD, 86, 103528

The COrE Collaboration et al. 2011, arXiv:1102.2181

Trotta, R., \& Melchiorri, A. 2005, PhRvL, 95, 011305

Viel, M., Haehnelt, M. G., \& Springel, V. 2010, JCAP, 6, 15

Wagner, C., Verde, L., \& Jimenez, R. 2012, ApL, 752, L31

Wang, X., Meng, X.-L., Zhang, T.-J., Shan, H., Gong, Y., Tao, C., Chen, X., \& Huang, Y. F. 2012, JCAP, 11, 18

Wong, Y. Y. Y. 2011, Annu. Rev. Nucl. Part. Sci., 61, 69

Xia, J.-Q., et al. 2012, JCAP, 6, 10

Zhao, G.-B., et al. 2012, arXiv:1211.3741 